九州大学学術情報リポジトリ

Kyushu University Institutional Repository

\title{
On an extension of the derivation relation for multiple zeta values
}

Kaneko, Masanobu

Faculty of Mathematics, Kyushu University

http://hdl. handle. net/2324/20462

出版情報: The Conference on L-Functions, pp.89-94, 2007. World Scientific バージョン：

権利関係 : 


\title{
On an extension of the derivation relation for multiple zeta values
}

\author{
Masanobu Kaneko
}

In this paper, we propose a conjectural generalization of the derivation relation for multiple zeta values. This extension was inspired by works of Alain Connes and Henri Moscovici on a certain Hopf algebra of transverse geometry [1], [2], and is thought of as a first attempt to materialize the suggestion given at the end of Section 7 in [4].

The multiple zeta value (MZV) is a real number defined for each index set $\mathbf{k}=\left(k_{1}, k_{2}, \ldots, k_{n}\right)$ of positive integers with $k_{1}>1$, by the convergent series

$$
\zeta(\mathbf{k})=\zeta\left(k_{1}, k_{2}, \ldots, k_{n}\right)=\sum_{m_{1}>m_{2}>\cdots>m_{n}>0} \frac{1}{m_{1}^{k_{1}} m_{2}^{k_{2}} \cdots m_{n}^{k_{n}}} .
$$

One of the fascinating features of these numbers is that the structures of (linear or algebraic) relations over $\mathbb{Q}$ among them reflect various other structures in mathematics and mathematical physics.

To describe the derivation relation of MZV's, we use the algebraic setup introduced by Hoffman [3]. Let $\mathfrak{H}=\mathbb{Q}\langle x, y\rangle$ be the non-commutative polynomial algebra over the rationals in two indeterminates $x$ and $y$, and $\mathfrak{H}^{1}$ and $\mathfrak{H}^{0}$ its subalgebras $\mathbb{Q}+\mathfrak{h} y$ and $\mathbb{Q}+x \mathfrak{h} y$, respectively. Let $Z: \mathfrak{H}^{0} \rightarrow \mathbb{R}$ be the $\mathbb{Q}$-linear map defined by $Z(1)=1$ and

$$
Z\left(x^{k_{1}-1} y x^{k_{2}-1} y \cdots x^{k_{n}-1} y\right)=\zeta\left(k_{1}, k_{2}, \ldots, k_{n}\right) .
$$

This "evaluation map" arises naturally from the iterated integral representation of MZV's. To find a linear relation among MZV's is to find an element in the kernel of the map $Z$.

For each integer $n \geq 1$, define the derivation $\partial_{n}$ on $\mathfrak{S}$ by

$$
\partial_{n}(x)=x(x+y)^{n-1} y, \quad \partial_{n}(y)=-x(x+y)^{n-1} y .
$$


Since $\mathfrak{H}$ is the polynomial algebra on $x$ and $y$, this uniquely determines the derivation $\partial_{n}$. (A derivation on $\mathfrak{H}$ is a linear map $\partial: \mathfrak{H} \rightarrow \mathfrak{H}$ satisfying Leibniz's rule $\partial(u v)=\partial(u) v+u \partial(v)$ for any $u, v \in \mathfrak{H}$.) Note that the derivation $\partial_{n}$ preserves $\mathfrak{H}^{1}$ and $\mathfrak{H}^{0}$. The derivation relation of MZV's is then the following.

Theorem. ([4, Corollary 6]) One has $Z\left(\partial_{n}(w)\right)=0$ for all $n \geq 1$ and all $w \in \mathfrak{H}^{0}$.

This relation supplies a large class of linear relations of MZV's. To add the derivation relation to (finite) double shuffle relation is equivalent to extend the double shuffle relation by using divergent MZV's. Also, the derivation relation is equivalent to Ohno's relation (minus duality). See [4] for the details.

We now proceed to generalize this. In [4, p.328], it is noticed that the derivation $\partial_{n}$ has an alternative description: Let $\theta$ be the derivation on $\mathfrak{H}$ defined by

$$
\theta(x)=x^{2}+(x y+y x) / 2, \quad \theta(y)=y^{2}+(x y+y x) / 2 .
$$

Then we have

$$
\partial_{n}=\operatorname{ad}(\theta)^{n-1}\left(\partial_{1}\right) /(n-1) !,
$$

where $\operatorname{ad}(\theta)(\partial):=[\theta, \partial]=\theta \partial-\partial \theta$. Let $H$ be the "Euler operator", namely, the linear endomorphism of $\mathfrak{H}$ which sends homogeneous elements $w$ to $\operatorname{deg}(w) w$.

Definition. Let $c$ be a rational number. For any $n \geq 1$, we define a linear endomorphism $\partial_{n}^{(c)}$ on $\mathfrak{H}$ by

$$
\partial_{n}^{(c)}=\operatorname{ad}\left(\theta^{(c)}\right)^{n-1}\left(\partial_{1}\right) /(n-1) !,
$$

where $\theta^{(c)}$ is the linear endomorphism defined by the rules

$$
\theta^{(c)}(x)=\theta(x), \quad \theta^{(c)}(y)=\theta(y)
$$

and

$$
\theta^{(c)}(u v)=\theta^{(c)}(u) v+u \theta^{(c)}(v)+c \partial_{1}(u) H(v)
$$

for any $u, v \in \mathfrak{H}$. 
Remark. This definition is modeled after the "twisted" Hopf algebra of Connes-Moscovici (see [1], [2], [4]).

Note that $\theta^{(0)}=\theta$ and $\partial_{n}^{(0)}=\partial_{n}$, and if $c \neq 0, \theta^{(c)}$ and $\partial_{n}^{(c)}$ are not derivations.

Conjecture 1. For all $n \geq 1, c \in \mathbb{Q}$, and $w \in \mathfrak{H}^{0}$, we have

$$
Z\left(\partial_{n}^{(c)}(w)\right)=0 .
$$

When we view $c$ as a parameter, we see that $\partial_{n}^{(c)}(w)$ is a polynomial in $c$ of degree $n-1$. Hence, the above conjecture is equivalent to the following.

Conjecture 1'. For all $n \geq 1$ and $i$ with $0 \leq i \leq n-1$ and $w \in \mathfrak{H}^{0}$, we have

$$
Z\left(\text { coefficient of } c^{i} \text { in } \partial_{n}^{(c)}(w)\right)=0 .
$$

We have checked this by Mathematica for all $n, i$ and $w$ with $n+\operatorname{deg}(w) \leq$ 9. Tatsushi Tanaka has extended the experiments by using Risa/Asir and verified the conjecture up to weight 12 . Below we give a table of various numbers of independent relations of MZV's obtained by $\partial_{n}^{(c)}$, detailed descriptions of the table being given in the remarks after the table. Recall the weight of the $\operatorname{MZV} \zeta\left(k_{1}, k_{2}, \ldots, k_{n}\right)$ is $k_{1}+k_{2}+\cdots+k_{n}$.

Table 1: (Conjectural) Numbers of Independent Relations

\begin{tabular}{c||cccccccccc} 
weight & 3 & 4 & 5 & 6 & 7 & 8 & 9 & 10 & 11 & 12 \\
\hline \hline$\partial_{n}(w)$ & 1 & 2 & 5 & 10 & 22 & 44 & 90 & 181 & 363 & 727 \\
\hline$\partial_{n}^{(c)}(w), c \neq-2$ fixed & 1 & 2 & 5 & 10 & 22 & 44 & 90 & 181 & 363 & 727 \\
\hline$\partial_{n_{1}}^{\left(c_{1}\right)}\left(w_{1}\right) \cup \partial_{n_{2}}^{\left(c_{2}\right)}\left(w_{2}\right)$ & 1 & 2 & 5 & 10 & 23 & 46 & 97 & 196 & 396 & \\
\hline$\partial_{n}^{(c)}(w)$ for all $c \cup$ duality & 1 & 2 & 5 & 10 & 23 & 46 & 98 & 200 & 413 & 838
\end{tabular}

Remarks. i) The first line of the data gives the number of linearly independent elements in $\mathfrak{H}$ among all $\partial_{n}(w)$ with $n \in \mathbb{N}$ and $w \in \mathfrak{H}^{0}$ varying under the condition $n+\operatorname{deg}(w)=$ weight. (The weight of each term of $Z\left(\partial_{n}(w)\right)$ is $n+\operatorname{deg}(w)$.)

ii) The second line is the same experiment with various fixed $c \in \mathbb{Q}$. The result says that, at least for tens of randomly chosen values of $c \neq-2$, 
the elements $\partial_{n}^{(c)}(w)$ as $n$ and $w$ vary give the same number of independent relations as $\partial_{n}(w)$, regardless of the choice of $c$. We have no proof of this (nor of $Z\left(\partial_{n}^{(c)}(w)\right)=0$, except in low weights). The reason why the value $c=-2$ is exceptional is explained by the identity $\partial_{n}^{(-2)}=(-1)^{n-1} \partial_{1}^{n}$, which, as Seidai Yasuda and Kentaro Ihara pointed out, is easily proved.

iii) The experiments of various single $c$ as in ii) may suggest that $\partial_{n}^{(c)}$ only gives the same set of relations as the original derivation $\partial_{n}$ does. However, we do have new relations, as the third line of the above table show. The third line of the table gives the number of linearly independent elements supplied by the union of $\partial_{n}^{\left(c_{1}\right)}(w)$ and $\partial_{n}^{\left(c_{2}\right)}(w)$ for randomly chosen different values of $c_{1}$ and $c_{2}(\neq-2)$. It is remarkable here again that any choice of $c_{1}$ and $c_{2}$ seems to give the same number of independent relations. This too is only experimental and is not yet proved.

iv) The final line is the result of taking all $c$ into account and moreover adding the duality relations. Up to weight 9 , we used the form of Conjecture $1^{\prime}$ to ensure the "for all $c$ " part, but from weight 10 on we only used the data for several rational values of $c$ for computational reason. Thus the number given may increase if we add more values of $c$, but we believe that does not occur, in light of Conjecture 2 below. The reason why we add the duality is also explained by Conjecture 2. If the duality is not added, the numbers obtained are strictly less at weights 11 and 12 (and probably for higher weights).

v) Seidai Yasuda pointed out the identity

$$
\theta^{(c)}=\theta^{(0)}+\frac{c}{2}\left(\theta^{(0)}-\theta^{(-2)}\right)
$$

or in general

$$
\theta^{(c)}=\theta^{(0)}-\frac{c}{c^{\prime}}\left(\theta^{(0)}-\theta^{\left(c^{\prime}\right)}\right)
$$

for any $c^{\prime} \neq 0$. Hence, to consider $\partial_{n}^{(c)}$ for all $c$ is also equivalent to consider $\operatorname{ad}\left(\theta^{\left(c_{1}\right)}\right) \operatorname{ad}\left(\theta^{\left(c_{2}\right)}\right) \cdots \operatorname{ad}\left(\theta^{\left(c_{n-1}\right)}\right)\left(\partial_{1}\right)$ with each $c_{i}=0$ and 1 (say).

Now let $a_{k}$ be the entry for weight $k$ of the last line of the above table (e.g., $a_{8}=46, a_{9}=98, a_{10}=200, \ldots$ ). Then, we have observed the following remarkable (conjectural) identity. 
Conjecture 2. For $k \geq 3$, we have

$$
2^{k-2}-a_{k}=\frac{1}{k-1} \sum_{d \mid k-1} \mu\left(\frac{k-1}{d}\right) 2^{d} .
$$

Here, $\mu$ is the Möbius function.

Remark. Let $L_{2}$ be the free graded Lie algebra of rank 2 over $\mathbb{Q}$ generated by $x$ and $y$ of degree 1 , and $L_{\infty}$ the free graded Lie algebra over $\mathbb{Q}$ with infinitely many generators $z_{i}(i=1,2,3, \ldots)$ whose degrees are given by $\operatorname{deg}\left(z_{i}\right)=i$. The number on the right-hand side of the conjecture is equal to the dimension of degree $k-1$ part of both $L_{2}$ and $L_{\infty}$, as Witt's formula shows. We note that the universal enveloping algebra of $L_{2}$ is identified with $\mathfrak{H}$, whereas that of $L_{\infty}$ is identified with $\mathfrak{H}^{1}$ under the identification $z_{i}=$ $x^{i-1} y$. (The algebra $\mathfrak{H}^{1}$ is freely generated by $z_{i}=x^{i-1} y, i=1,2,3, \ldots$.) This observation suggests intimate connections between these Lie algebras and the relations of MZV's. Note that the number $2^{k-2}$ on the left is the total number of MZV's of weight $k$. Thus the number on the left-hand side of the conjecture is the maximal number of independent MZV's, under the conjectural relations $Z\left(\partial_{n}^{(c)}(w)\right)=0$ and the duality.

The most curious feature of Conjecture 2 is that the weights concerned on both sides differ by 1 . The conjecture implies the possibility of constructing linearly independent (under the conjectural relations and the duality) elements of MZV's of weight $k$ from a basis of homogeneous part of degree $k-1$ of $L_{2}$ or $L_{\infty}$. But how to construct such elements? We tried several ways at random and found one way (using the derivation $\delta_{y}$ in [4, $\S 4]$ and the regularization) which bears the numerical test up to weight 13 , but there is no theoretical support to believe that is the right way. It is most desirable to prove (or disprove) this conjecture and clarify the suggested connection of the MZV to the Lie algebras $L_{2}$ and $L_{\infty}$.

Acknowledgment. The author would like to thank Seidai Yasuda at RIMS, Kyoto, for valuable comments and suggestions.

\section{References}

[1] Connes, A. and Moscovici, H., Modular Hecke algebras and their Hopf symmetry, Moscow Mathematical Journal 4 (2004), 67-109. 
[2] Connes, A. and Moscovici, H., Rankin-Cohen brackets and the Hopf algebra of transverse geometry, Moscow Mathematical Journal 4 (2004), 111-130.

[3] Hoffman, M., The algebra of multiple harmonic series, J. Algebra 194 (1997), 477-495.

[4] Ihara, K., Kaneko, M., and Zagier, D., Derivation and double shuffle relations for multiple zeta values. Compositio Math. 142-02 (2006), 307-338.

Email: mkaneko@math.kyushu.u-ac.jp

Faculty of Mathematics, Kyushu University 33,

Fukuoka 812-8581, Japan. 